\title{
Simulation of Single Molecule Inelastic Electron Tunneling Signals in Paraphenylene-Vinylene Oligomers and Distyrylbenzene[2.2]paracyclophanes
}

\author{
Jeremy B. Maddox, ${ }^{*}, \dagger$ Upendra Harbola, ${ }^{\dagger}$ Ning Liu,,$\$$ Christophe Silien,,$\#$ Wilson Ho,, \\ Guillermo C. Bazan," and Shaul Mukamel ${ }^{\dagger}$ \\ Department of Chemistry, University of California, Irvine, California 92697, and Department of Physics, \\ University of California, Irvine, California 92697, and Department of Chemistry, University of California, \\ Santa Barbara, California 93106
}

Received: March 14, 2006

\begin{abstract}
Inelastic resonances in the electron tunneling spectra of several conjugated molecules are simulated using the nonequilibrium Greens function formalism. The vibrational modes that strongly couple to the electronic current are different from the infrared and Raman active modes. Spatially resolved inelastic electron tunneling (IET) intensities are predicted. The simulated IET intensities for a large distyrylbenzene paracyclophane molecule are in qualitative agreement with recent experimental results.
\end{abstract}

\section{Introduction}

Inelastic electron tunneling spectroscopy (IETS) has long been used to characterize organic dopants in metal-insulator-metal junctions. ${ }^{1}$ Interactions between tunneling electrons and molecules adsorbed on an insulating layer open up inelastic scattering channels for charge transport via the excitation of molecular vibrational or electronic transitions. These give rise to nonlinear current versus voltage $(I / V)$ characteristics that occur when the applied bias energy exceeds a vibrational or electronic transition energy $e|V| \geq \hbar \Omega$ and show up as discrete steps in the conductance $\mathrm{d} I / \mathrm{d} V$ that translate to peaks in the $\mathrm{d}^{2} I / \mathrm{d} V^{2}$ curve. Early IETS work emphasized its chemical sensitivity, where strong well-resolved signals could be obtained over a very broad $(0-3 \mathrm{eV})$ spectral range covering vibrational and electronic resonances from submonolayer concentrations of adsorbate at metal-oxide interfaces. Studies involving catalysis, chemisorption of organic acids on alumina, and probing radiative damage in biological molecules are among the frequently highlighted examples of IETS. $^{2}$ Perhaps one of the most noteworthy features of vibrational IETS is that both IR and Raman active modes are observed with more or less equivalent intensities. ${ }^{3}$ Factors such as thermal effects and electron-phonon coupling, which affect IETS peak intensities and line-shapes are the subject of ongoing research that has fundamental implications for understanding electron transport. ${ }^{4}$ In more recent years, IETS measurements have been employed to characterize the conductance properties of self-assembled organic monolayers (SAMs) directly chemisorbed on metallic leads in metalmolecule-metal junctions. ${ }^{5-12}$

Over the past decade, technological advancements pertaining to the measurement of electronic transport through single molecules have engendered a diverse array of experimental and

\footnotetext{
Department of Chemisty, University of California, Irvine.

Department of Physics, University of California, Irvine.

$\S$ Present address: Surface Science Research Centre, University of Liverpool, Liverpool L69 3BX, U.K.

\# On leave from Laboratoire de Spectroscopie Moléulaire de Surface, Faculté Universitaires Notre-Dame de la Paix, 61 rue de Bruxelles, B-5000 Namur, Belgium.

"Department of Chemistry, University of California, Santa Barbara.
}

theoretical studies. These include charge transport through single-molecule break junctions, ${ }^{13-15}$ chemisorption and binding configurations, ${ }^{16,17}$ chemical reactions at interfaces, ${ }^{18-20}$ current induced dynamics, ${ }^{21-25}$ and single-molecule adsorbates in scanning tunneling microscopy (STM) where the inelastic current can be resolved both spatially and spectrally. ${ }^{26-35}$

Inelastic effects in single molecules are particularly intriguing because they can be observed without the loss of information associated with ensemble averaging. ${ }^{36}$ Local environmental factors, such as binding configurations and orientational dependencies can be explored. This is crucial to the development of single-molecule electronic devices. For example, the formation of polarons ${ }^{37}$ has been suggested as one possible mechanism for the negative resistance observed in some molecular wires. $6,7,38-40$ Junction heating ${ }^{41-44}$ and reversible conformational changes associated with hysteretic molecular switching, ${ }^{45}$ are more obviously related to electron-phonon coupling in the junction. IET effects may be selectively enhanced or suppressed through the judicious synthetic manipulation of molecular functional groups, thus leading to tailored $I / V$ device properties.

In this paper, we present a theoretical investigation of electron-phonon interactions in IET in two families of conjugated molecular chromophores: paraphenylene-vinylene oligomers (OPV, Figure 1) and distyrylbenzene paracyclophanes (DBP, Figure 2). Chromophores derived from OPVn's are frequently used as model systems for PPV semiconducting materials which are well-known for their bulk electrooptical properties. ${ }^{46}$ DBP represents an interesting class of compounds with bi-chromophoric interactions between two optically active species held in close proximity with a well-defined orientation. ${ }^{47}$ The configuration of the distyrylbenzene groups imitates the crossing of two molecular wires that can communicate with one another electronically through the $\pi$-stacked orbitals in the paracyclophane core. The larger OPV and DBP molecules are divided into smaller species and the IETS resonances of the individual benzene, styryl, and paracyclophane groups are examined. We then study how the IET signal changes when the various components are put together. Our approach, based on a perturbative expansion of the molecular electronic Greens function in electron-phonon coupling, is outlined in section 2. 


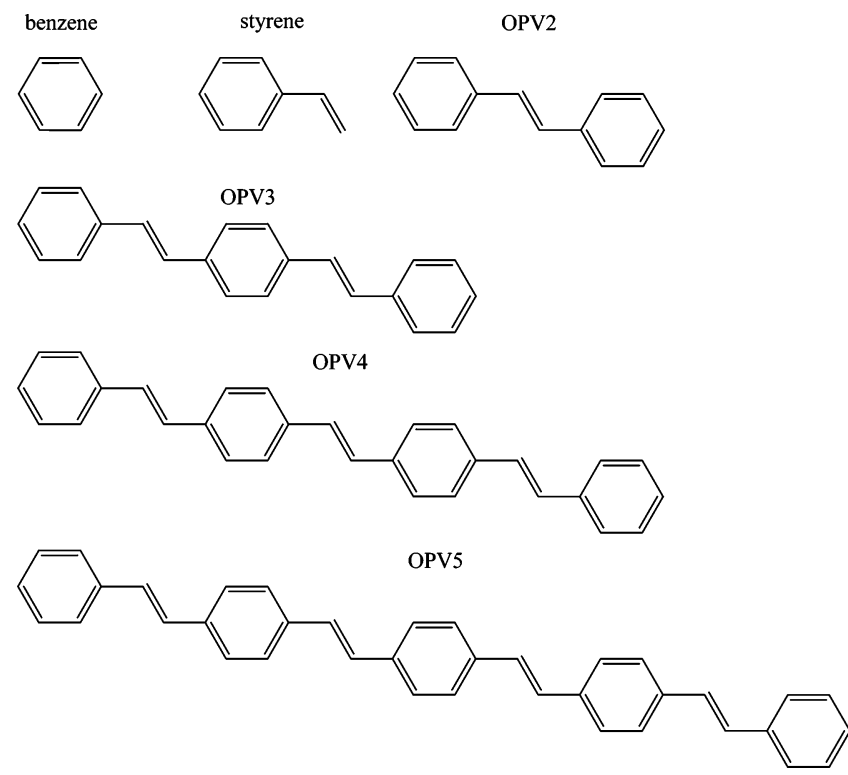

Figure 1. Benzene, styrene, and a family of paraphenylene-vinylene oligomers (OPVn's) considered in this study.

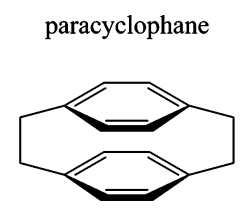

trans-2Rd

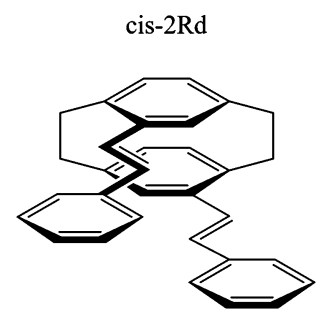

$3 R d$
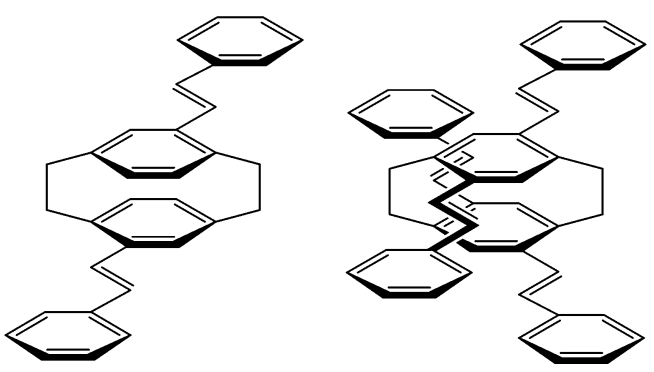

Figure 2. Paracyclophane and a family distyrylbenzene paracyclophane (DBP) derivatives considered in this study.

In section 3 we discuss the numerical procedure and applications are presented in section 4 . Comparison with experiment is made where applicable. Finally, in section 5 we conclude with a brief summary.

\section{Theory}

The nonequilibrium Greens function (NEGF) formalism ${ }^{48-51}$ provides an exact prescription for calculating molecular currents, whether elastic or inelastic. The procedure involves an iterative (self-consistent) calculation of the electronic and phonon selfenergies and Greens functions, which yields the exact charge distribution for the nonequilibrium system subjected to an external bias under open boundary conditions. This method has been widely applied to elastic currents through many types of molecular junctions. ${ }^{52-54}$ Recently, it was extended to inelastic currents in model systems ${ }^{55-57}$ and in small molecules such as benzenedithiolate. ${ }^{58}$ Calculating inelastic currents through a molecular junction requires the self-consistent calculation for the electronic self-energy at each bias value; each iteration involves a separate self-consistent calculation of the phonon selfenergy that, in principle, requires a re-optimization of the atomic coordinates, calculation of the Hessian, and evaluation of the electron-phonon coupling matrix elements. So far, this computationally expensive procedure has not been implemented for large molecules.

A perturbative expansion of the molecular electronic Greens functions in the electron-phonon coupling ${ }^{59,60}$ can be readily applied to large molecules. We consider a single molecule coupled to two leads that provide the source and drain for electrons and may be taken as either a metallic surface or an STM probe. We use Latin $(i, j, \ldots)$ and $\operatorname{Greek}(\mu, \eta, \ldots)$ indicies to denote molecule and tip orbitals, respectively. The steadystate current through the junction at the tip-molecule contact is given by ${ }^{61}$

$$
\begin{aligned}
I=-\frac{\mathrm{i} e}{\hbar} \sum_{i j \mu \eta} \rho_{\mu \eta} V_{i \mu} V_{\eta j}^{\dagger} \int \mathrm{d} E\left[f(E+e V) G_{j i}^{>}(E)+\right. \\
\left.(1-f(E+e V)) G_{i j}^{<}(E)\right]
\end{aligned}
$$

where $\rho_{\mu \eta}$ is the lead's density of states (assumed to be independent of energy), $V_{i \mu}$ is the molecule/lead electronic coupling matrix element, and $f(E)$ is the Fermi-Dirac distribution function. The "greater" and "lesser" $G^{>}(E) / G^{<}(E)$ Greens functions represent electron transfer from/to an occupied/ unoccupied orbital of the lead to/from an unoccupied/occupied orbital of the molecule, respectively. The difference between these two propagators gives the net electronic flux at the contact. A similar expression can be written for the current across the other contact. At steady state, the current through both contacts is the same but opposite in sign and can be combined to give a more symmetric expression of the total current. ${ }^{62}$ At zero temperature, the differential conductance is given by

$$
\frac{\mathrm{d} I}{\mathrm{~d} V}=\frac{e^{2}}{\hbar} \sum_{i j \mu \eta} \rho_{\mu \eta} V_{i \mu} V_{\eta j}^{\dagger} F_{i j}\left(\epsilon_{\mathrm{F}}-e V\right)
$$

where

$$
F_{i j}(E) \equiv-\mathrm{i}\left[G_{j i}^{>}(E)-G_{i j}^{<}(E)\right]
$$

represents the net flux of electrons between a lead and molecule that includes all elastic and inelastic scattering channels. Using the Dyson equations, ${ }^{63}$ we obtain a matrix expression for $F(E)$

$$
F=2 \pi\left(\rho_{0}+2 \rho_{0} \operatorname{Re}\left[\Sigma^{\mathrm{r}} G^{\mathrm{r}}\right]+G^{r} \Sigma^{r} \rho_{0} \Sigma^{\mathrm{a}} G^{\mathrm{a}}-\frac{1}{\pi} G^{\mathrm{r}} \operatorname{Im}\left[\Sigma^{\mathrm{r}}\right] G^{\mathrm{a}}\right)
$$

where all terms are energy dependent matrices in the single electron (molecular orbital) space; $\rho_{0}(E)$ is the molecular density of states (including the interaction with the leads but without the influence of phonons); and $G^{\mathrm{r}}(E) / G^{\mathrm{a}}(E)$ are the nonequilibrium retarded/advanced Greens functions whose self-energies $\Sigma^{\mathrm{r}}(E) / \sum^{\mathrm{a}}(E)$ encompass the effects of the electron-phonon interactions and depend on both electron and phonon Greens functions. ${ }^{56,63}$ The real part of the self-energy is usually small and is a smooth function of energy that shifts the molecular orbital energies but does not affect their line-widths. ${ }^{64}$ Neglecting the real part and assuming that the phonons are at equilibrium, the retarded self-energy to lowest order in the electron-phonon coupling is given by

$$
\begin{aligned}
\Sigma_{0}^{\mathrm{r}}(E) \approx-\mathrm{i} \pi \sum_{m} \lambda^{m} \rho_{0}\left(E+\hbar \Omega_{m}\right) \lambda^{m} \theta\left(E-\epsilon_{\mathrm{F}}+\hbar \Omega_{m}\right)+ \\
\lambda^{m} \rho_{0}\left(E-\hbar \Omega_{m}\right) \lambda^{m} \theta\left(E-\epsilon_{\mathrm{F}}-\hbar \Omega_{m}\right)
\end{aligned}
$$


where $\lambda^{m}$ is the electron-phonon coupling matrix for the $m$ th molecular vibration with frequency $\Omega_{m}$. The step functions $\theta(E)$ in eq 4 originate from taking the zero temperature limit of the Fermi-Dirac distribution function for the lead; these discrete steps are responsible for the inelastic discontinuities in $\mathrm{d} I / \mathrm{d} V$ and their magnitude is scaled by the coupling matrices.

Expanding the nonequilibrium Greens functions

$$
\begin{aligned}
& G^{\mathrm{r}}=G_{0}^{\mathrm{r}}+G_{0}^{\mathrm{r}} \Sigma_{0}^{\mathrm{r}} G_{0}^{\mathrm{r}}+\cdots \\
& G^{\mathrm{a}}=G_{0}^{\mathrm{a}}+G_{0}^{\mathrm{a}} \Sigma_{0}^{\mathrm{a}} G_{0}^{\mathrm{a}}+\cdots
\end{aligned}
$$

in eq 4, we obtain the following zeroth- and second-order terms in the electron-phonon coupling

$$
\begin{gathered}
F^{(0)}=2 \pi \rho_{0} \\
F^{(2)}=2 \pi \rho_{0} \operatorname{Im}\left[\Sigma_{0}^{\mathrm{r}}\right] \rho_{0}-\frac{1}{\pi} G_{0}^{\mathrm{r}} \operatorname{Im}\left[\Sigma_{0}^{\mathrm{r}}\right] G_{0}^{\mathrm{a}}
\end{gathered}
$$

The equilibrium density of states, Greens functions, and selfenergies are expanded as a sum over molecular orbitals

$$
\begin{gathered}
\rho_{0}(E)=\sum_{k}|k\rangle\langle k| \delta\left(E-\epsilon_{k}\right) \\
G_{0}^{\mathrm{r}}(E)=\sum_{k} \frac{|k\rangle\langle k|}{E-\epsilon_{k}+\mathrm{i} \eta_{k}} \\
\sum_{0 i j}^{\mathrm{r}}(E)=-\mathrm{i} \pi \sum_{m k} \lambda_{i k}^{m} \lambda_{k j}^{m}\left[\delta\left(E-\epsilon_{k}+\hbar \Omega_{m}\right) \theta\left(\epsilon_{\mathrm{F}}-E-\hbar \Omega_{m}\right)+\right. \\
\left.\delta\left(E-\epsilon_{k}-\hbar \Omega_{m}\right) \theta\left(E-\epsilon_{F}+\hbar \Omega_{m}\right)\right]
\end{gathered}
$$

where $\eta_{i}$ is the broadening of the $i$ th molecular orbital due to the interaction with the surface and the tip. This gives

$$
\begin{gathered}
F_{i j}^{(0)}(E)=2 \pi \delta_{i j} \delta\left(E-\epsilon_{i}\right) \\
F_{i j}^{(2)}(E)=\sum_{m} Q_{i j+}^{m}(E) \theta\left(\epsilon_{\mathrm{F}}-E-\hbar \Omega_{m}\right)+ \\
Q_{i j-}^{m}(E) \theta\left(E-\epsilon_{\mathrm{F}}-\hbar \Omega_{m}\right)
\end{gathered}
$$

where

$$
\begin{gathered}
Q_{i j \pm}^{m}(E)=-2 \pi^{2} \sum_{k} \lambda_{i k}^{m} \lambda_{k j}^{m} \delta\left(E-\epsilon_{i}\right) \delta\left(E-\epsilon_{j}\right) \delta\left(E-\epsilon_{k} \pm \hbar \Omega_{m}\right)+ \\
\sum_{k} \frac{\lambda_{i k}^{m} \lambda_{k j}^{m} \delta\left(E-\epsilon_{k} \pm \hbar \Omega_{m}\right)}{\left(E-\epsilon_{i}+\mathrm{i} \eta_{i}\right)\left(E-\epsilon_{j}-\mathrm{i} \eta_{j}\right)}
\end{gathered}
$$

Assuming that the zeroth-order (elastic) contribution to the conductance is constant over the applied bias range, gives for the inelastic conductance

$$
\begin{array}{r}
\frac{\mathrm{d} I}{\mathrm{~d} V}=\frac{2 \pi e^{2}}{\hbar} \sum_{i j \mu \eta} \rho_{\mu \eta} V_{i \mu} V_{\eta j}^{\dagger} \sum_{m}\left(Q_{i j+}^{m}\left(\epsilon_{\mathrm{F}}-e V\right) \theta\left(e V-\hbar \Omega_{m}\right)+\right. \\
\left.Q_{i j-}^{m}\left(\epsilon_{\mathrm{F}}-e V\right) \theta\left(-e V-\hbar \Omega_{m}\right)\right)
\end{array}
$$

Furthermore, because the energy variation of the coefficients $Q_{i j \pm}^{m}(E)$ is smooth compared to step functions $\theta(E)$, the second derivative of the current shows peaks at bias energies resonant with the vibrational transition energies $e V= \pm \hbar \Omega_{m}$

$$
\begin{array}{r}
\frac{\mathrm{d}^{2} I}{\mathrm{~d} V^{2}}=\frac{2 \pi e^{3}}{\hbar} \sum_{i j \mu \eta} \rho_{\mu \eta} V_{i \mu} V_{\eta j}^{\dagger} \sum_{m}\left(Q_{i j+}^{m}\left(\epsilon_{\mathrm{F}}-e V\right) \delta\left(e V-\hbar \Omega_{m}\right)-\right. \\
\left.Q_{i j-}^{m}\left(\epsilon_{\mathrm{F}}-e V\right) \delta\left(-e V-\hbar \Omega_{m}\right)\right)
\end{array}
$$

In general, the amplitude of the steps/peaks can be positive or negative at either polarity, depending on the sign of the $Q_{i j \pm}^{m}\left(\epsilon_{\mathrm{F}} \pm \hbar \Omega_{m}\right)$ factors.

Equations 15 and 16 are our final expressions for the inelastic conductance and the IETS intensity, respectively. We next describe the technical details of our computational procedure for evaluating the tip-molecule and electron-phonon coupling matrices which are the input parameters for these expressions.

\section{Numerical Procedure}

Predicting the energetic line-up of the molecular orbitals relative to the Fermi level and the line widths of the molecular orbital energies relative to density of states of the leads requires the precise electronic structure of the contact region. These factors are crucial for predicting absolute magnitudes of the elastic and inelastic contributions to the current through a molecular junction but are less important for comparing different inelastic channels that primarily involve only the molecular degrees of freedom. In this study, we neglect the details of the electrodes' electronic structure and contact geometry and focus on the inelastic contributions to the current arising from the internal vibrations of isolated molecules. Obviously, this precludes the examination of modes involving the electrode atoms and translational ("bouncing") and librational motion of the molecule relative to the contacts. Electric field effects and the explicit electronic coupling between the leads and the molecule, neglected in this study, are also expected to affect the absolute value of IETS resonance intensities.

First, the ground-state energy of an isolated molecule is minimized by optimizing the nuclear geometry. A normal-mode analysis is performed to calculate the Hessian matrix, which is diagonalized to obtain the vibrational frequencies and atomic displacement vectors. All electronic structure calculations are preformed with the Gaussian 03 package ${ }^{65}$ at the HF/3-21G level. The line widths of the molecular energy levels are free parameters in our model. We assume that the Fermi energy $\epsilon_{\mathrm{F}}$ lies between the highest occupied and lowest unoccupied molecular orbitals (HOMO/LUMO); otherwise the molecule will become charged, thus changing the occupancy and energy spacing of the orbitals. The Fermi level is set at the midpoint between the HOMO and LUMO. Lorentzian broadening of the molecular orbitals and phonon transition energies due to the interaction with the leads is included by making the substitutions

$$
\begin{gathered}
\delta\left(E-\epsilon_{i}\right) \rightarrow \frac{1}{\pi} \frac{\eta_{i} / 2}{\left(E-\epsilon_{i}\right)^{2}+\eta_{i}^{2} / 4} \\
\delta\left(E-\hbar \Omega_{m}\right) \rightarrow \frac{1}{\pi} \frac{\eta_{m} / 2}{\left(E-\hbar \Omega_{m}\right)^{2}+\eta_{m}{ }^{2} / 4}
\end{gathered}
$$

everywhere in eqs $9-16$. We uniformly set $\eta_{i}=1.0 \mathrm{eV}$ for all orbital energies and $\eta_{m}=0.01 \mathrm{eV}$ for the molecular vibrational energies. 
The electronic coupling matrix elements $V_{i \mu}$ depend primarily on the junction configuration. Typically, for molecular wire junctions, an extended molecular system is defined that contains the conducting molecule and several layers of the leads' periodic lattice structure. ${ }^{66}$ The electrode portion of the extended molecule is taken to be sufficiently large so that the electronic coupling elements $V_{i \mu}$ between the extended system and the semi-infinite leads are identical to the Hamiltonian matrix elements between adjacent unit cells of the bulk electrode. In STM junctions, the tip's position, and hence the electronic coupling, is experimentally controlled. The simplest weakcoupling approach for calculating electronic coupling follows from Tersoff-Hamann (TH) theory, ${ }^{67,68}$ where the STM probe is represented by a spherical potential well centered about the tip's position $\mathbf{r}_{0}$. The tip electronic structure is reduced to a single s-type orbital $\left|\mathbf{r}_{0}\right\rangle$ at the Fermi energy $\epsilon_{\mathrm{F}}$ and the electronic coupling elements are proportional to the overlap of $\left|\mathbf{r}_{0}\right\rangle$ with the molecular orbitals of the substrate

$$
V_{i \mu} \approx \delta_{\mu \mathbf{r}_{0}} V_{i \mathbf{r}_{0}} \propto \delta_{\mu \mathbf{r}_{0}}\left\langle\mathbf{r}_{0} \mid i\right\rangle \equiv \delta_{\mu \mathbf{r}_{0}} V_{i}\left(\mathbf{r}_{0}\right)
$$

Using this approximation and taking only the zeroth-order term $F^{(0)}(E)$ in eq 2 gives the TH result for conductance commonly used to simulate topographic STM images of surfaces and molecular adsorbates. ${ }^{67,68} F^{(2)}(E)$ is Lorente and Perrson's generalization of $\mathrm{TH}$ theory that also includes the lowest (second-) order inelastic contributions to the conductance due to the interaction with phonons; this approach has been used to simulate STM-IETS topographic images of vibrational resonances. ${ }^{34,59,60}$ In our calculations, the electronic couplings matrix elements $V_{i}\left(\mathbf{r}_{0}\right)$ are computed by introducing a ghost atom centered at the desired tip position $\mathbf{r}_{0}$ with a single $1 \mathrm{~s}$ orbital using parameters taken from the 3-21G basis set for silver atoms. The atomic overlap integrals $\left\langle\mathbf{r}_{0} \mid \alpha\right\rangle$ with the molecule's atomic basis functions $|\alpha\rangle$ are calculated so that the overlap coupling to the $i$ th molecular orbital is given by

$$
V_{i}\left(\mathbf{r}_{0}\right) \equiv\left\langle\mathbf{r}_{0} \mid i\right\rangle=\sum_{\alpha} c_{i \alpha}\left\langle\mathbf{r}_{0} \mid \alpha\right\rangle
$$

where $c_{i \alpha}$ are the molecular orbital coefficients. STM spatial maps are simulated by including a grid of ghost atomic centers positioned above the molecule and properly oriented with respect to a surface of interest. One can further simplify this by assuming that the tip has perfect spatial resolution $\left\langle\mathbf{r} \mid \mathbf{r}_{0}\right\rangle \approx$ $\delta\left(\mathbf{r}-\mathbf{r}_{0}\right)$. The coupling elements then simply become the value of the orbital wave function $\psi_{i}(\mathbf{r})$ evaluated at the tip's position, $V_{i}\left(\mathbf{r}_{\mathrm{o}}\right) \approx \psi_{i}\left(\mathbf{r}_{0}\right)$. Using this approximation in eq 16 and integrating over $\mathbf{r}_{0}$ removes the spatial dependence from the IETS signal and is useful for making general comparisons with optical IR and Raman spectra.

To calculate the inelastic signal, we must also evaluate the electron-phonon coupling matrices $\lambda^{m}$ for each molecular vibration $m$. In the atomic orbital basis representation, the electron-phonon coupling matrix elements are given by ${ }^{69}$

$$
\lambda_{\alpha \beta}^{m}=\sum_{n} \sqrt{\frac{\hbar}{2 M_{n} \Omega_{m}}} \Delta_{n m} \cdot\left\langle\alpha\left|\nabla_{X_{n}} H\right| \beta\right\rangle
$$

where the sum runs over all atoms, $M_{n}$ is the atomic weight of the $n$th atom, $\Delta_{n m}$ is the mass-weighted dimensionless displacement vector of $n$th atom for the $m$ th normal mode, and $\nabla_{X_{n}} H$ is the gradient of the electronic Hamiltonian with respect to the Cartesian coordinates of the $n$th atom at the equilibrium nuclear
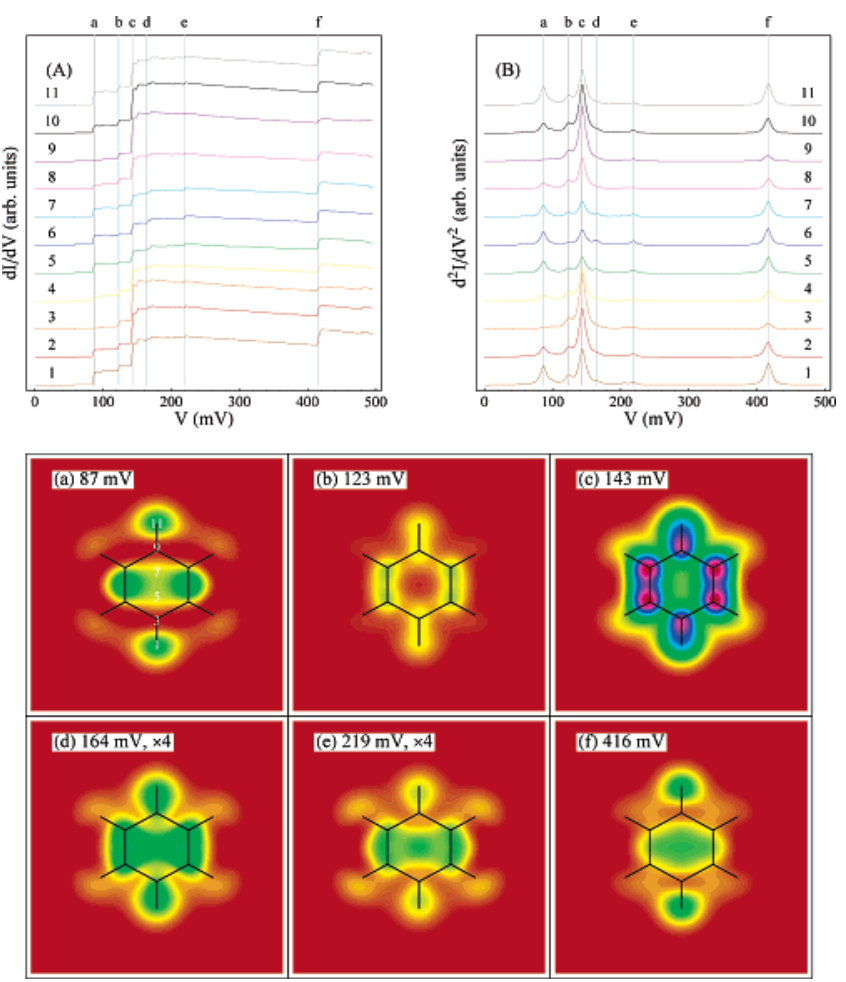

Figure 3. (A) Calculated conductance curves, eq 15, for benzene at eleven different tip positions; the curves are vertically displaced for visual clarity. The tip's height is held constant at $1 \AA$ above the molecular plane whereas the lateral position is varied as depicted by the white numbers (even numbers are omitted) in (a) where the solid black lines represent the molecular structure of benzene. (B) Calculated IETS signals, eq 16, at different positions. The vertical gray lines, labeled $\mathrm{a}-\mathrm{f}$, in (A) and (B) indicate the spectral positions of the six most intense inelastic contributions to the current. The overall intensity of the IETS signal and the relative intensities of the peaks are sensitive to the spatial position of the tip. (a)-(f) Spatial maps of these IETS peak intensities as a function of the tip's lateral position (height is constant). The molecular structure is superimposed as a visual reference and the less intense peaks have been scaled by a factor of 4 to improve the contrast.

configuration. Previously, the electron-phonon coupling matrices were evaluated numerically by displacing atoms along a normal coordinate and calculating the finite difference derivatives of the molecular orbitals and energies. ${ }^{59}$ These derivatives are then related to the coupling elements via first-order perturbation theory. In this work, the gradient of the Hamiltonian in the atomic orbital basis is obtained analytically by solving the coupled perturbed Hartree-Fock (CPHF) equations as implemented in Gaussian 03. The $\lambda^{m}$ matrices eq 21 are then calculated and transformed into the molecular orbital basis representation.

Using the ingredients described above, the inelastic conductance eq 15 and the IETS intensity eq 16 were calculated as a function of tip position and applied bias. The results are presented in the next section.

\section{Results and Discussion}

Benzene, Styrene, and OPVn. Our HF/3-21G calculation for benzene gives the planar $D_{6 h}$ conformation with doubly degenerate HOMO $\left(\mathrm{E}_{1 \mathrm{~g}}\right)$ and LUMO $\left(\mathrm{E}_{2 \mathrm{u}}\right)$ orbitals. Panels A and $\mathrm{B}$ of Figure 3 show the conductance and its derivative as a function of the applied bias for eleven tip positions; the curves are vertically displaced for visual clarity. The s orbital representing the STM tip is placed $1 \AA$ above the molecular plane and 
the lateral tip position is indicated by the white numbers shown in Figure 3a, where the black lines represent the molecular structure. The labels for the even-numbered positions are omitted for clarity. The inelastic conductance profiles exhibit a sequence of discontinuous steps occurring when the applied bias energy becomes resonant with a vibrational mode. The magnitude of the step at a given resonance is determined by the strength of the electron-phonon and tip-molecule coupling elements. The vertical gray lines labeled $\mathrm{a}-\mathrm{f}$ indicate the spectral positions of the six most intense peaks. We note that all the vibrational modes contribute to the IETS signal; there are no apparent selection rules as in IR and Raman spectroscopies. The intensity of the contribution from a given mode depends on how strongly it couples to a given orbital and the coupling of the molecular orbital to the tip orbital. The latter varies with the tip position and the IETS intensity for a given peak exhibits an interesting spatial pattern that provides information about the distribution of charge density that couples to the molecular vibrations. The bottom panels show the spatial profiles of the IETS intensity corresponding to the peaks labeled in the top panels.

The vibrational modes contributing to these peaks are identified using Wilson-Vársanyi nomenclature. ${ }^{70,71}$ (a) involves the radial skeletal stretching mode $6 \mathrm{~b}$ at $87 \mathrm{mV}$ and shows enhanced intensity centered over two of the carbon bonds and two of the hydrogen atoms. We note that the degenerate mode $6 \mathrm{a}$ is not as strongly coupled as $6 \mathrm{~b}$. (b) and (c) involve several different types of modes but the strongest contribution for both of these peaks comes from out-of-plane $\mathrm{C}-\mathrm{H}$ bending vibrations; these are (b) modes $10 \mathrm{a}$ and $10 \mathrm{~b}$ at $123 \mathrm{mV}$ and (c) modes $17 \mathrm{a}$ and $17 \mathrm{~b}$ at $143 \mathrm{mV}$. Overall, modes $17 \mathrm{a}$ and $17 \mathrm{~b}$ are the most strongly coupled vibrations and the IETS intensity is most intense near the carbon atoms. The peak (d) at $164 \mathrm{mV}$ is very weak and comes from the degenerate in-plane $\mathrm{C}-\mathrm{H}$ bending modes 9a and 9b. (e) at $219 \mathrm{mV}$ is due the ring stretching modes $8 \mathrm{a}$ and $8 \mathrm{~b}$; these are weakly coupled to the current and have small contributions to the IETS intensity. All of the $\mathrm{C}-\mathrm{H}$ stretching modes contribute to peak (f) at $416 \mathrm{mV}$; however, mode $7 \mathrm{~b}$ makes a stronger contribution.

The IET spectrum of benzene has been studied extensively with low-temperature STM: Lauhon and $\mathrm{Ho}^{72,73}$ and separately Komeda and co-workers ${ }^{74}$ have utilized STM-IETS to examine the dehydrogenation of benzene on copper surfaces. Neither group reports any IETS resonance for benzene; however, the dehydrogenated benzene fragment does exhibit $\mathrm{C}-\mathrm{H}$ stretching peaks. Pascual and co-workers ${ }^{31}$ have measured the three lowfrequency adsorbate-surface modes of benzene on $\mathrm{Ag}(110)$ but did not observe internal vibrational resonances. The experiment of Reed and co-workers ${ }^{5}$ involved $I / V$ measurements of benzenedithiolate (BDT) SAMs in a molecular break junction, but the IET vibrational spectra were not reported. Recently, however, there have been several theoretical studies using selfconsistent NEGF methods to simulate the IET spectrum of BDT attached to gold electrodes. Asai ${ }^{58,75}$ reports that the most strongly coupled vibration at $1240 \mathrm{~cm}^{-1}$ has $\mathrm{A}_{\mathrm{g}}$ symmetry and appears to be consistent with mode 1 . Sergueev and co-workers ${ }^{69}$ have examined the electron-phonon coupling strengths in BDT at low and high bias values. At $1 \mathrm{~V}$ they find that the centerof-mass vibrations $(8-14 \mathrm{mV})$ contribute the strongest, whereas at bias values $<0.5 \mathrm{~V}$ a low-frequency librational mode and internal modes $\mathrm{A}_{\mathrm{g}}(4)$ (mode 1 ) and $\mathrm{B}_{1 \mathrm{u}}$ (mode 19a) are more strongly coupled. The two internal modes have frequencies at roughly $1375-1400 \mathrm{~cm}^{-1}$. We note that in our calculations the vibrational modes and electron-phonon couplings were computed at zero bias.
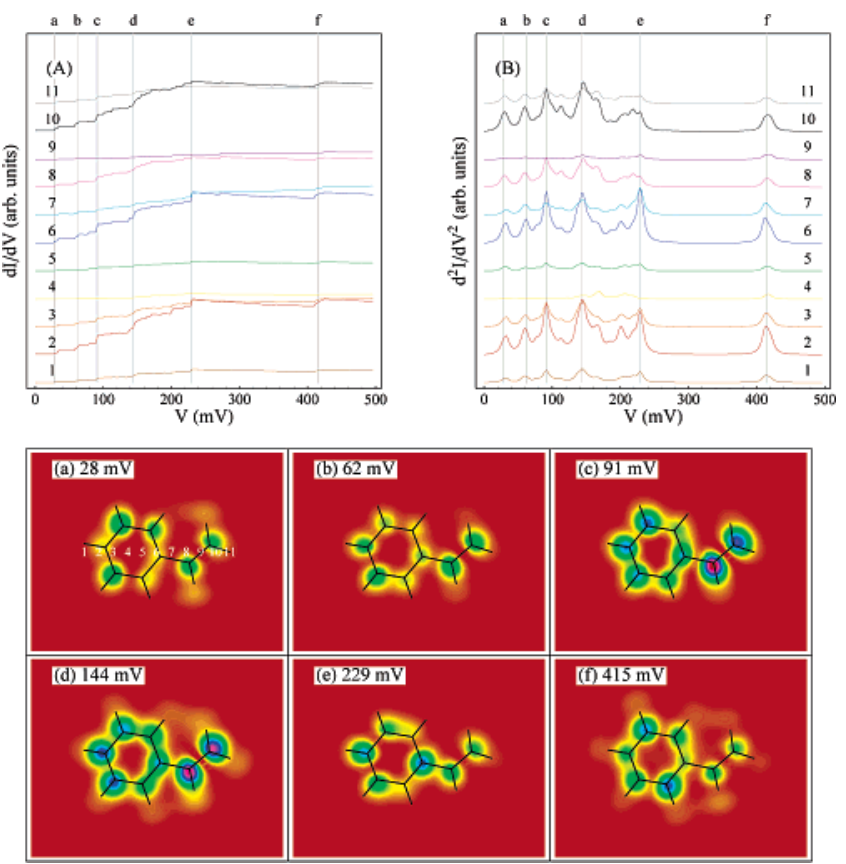

Figure 4. (A) and (B) calculated conductance and IETS intensities for styrene at different tip positions, depicted in (a). (a)-(f) Spatial maps of the most intense IETS peaks. See the caption in Figure 3 and text for more details.

For styrene and OPVn's there is some difficulty in determining the precise minimum energy conformation. Discrepancies between experiment and calculations (and between different levels of theory) concerning the planarity in these species stem form the notoriously flat potential surface involving torsional rotation about $\mathrm{C}-\mathrm{C}$ bonds joining the phenyl and vinyl groups. ${ }^{76}$ In our HF/3-21G calculations we impose a planar configuration; thus, there are some imaginary frequencies associated with the torsional motion; we ignore these modes in the calculation of the conductance and IETS signals. Panels A and B of Figure 4 show the simulated conductance and IETS intensities as a function of the applied bias, respectively. The eleven curves correspond to the tip positions labeled by white numbers in Figure 4a. The bottom panels correspond to the spatially resolved IETS intensity at a constant bias voltage sliced at $1 \AA$ above the molecular plane.

The IETS spectra of styrene show the ring modes in benzene with additional contributions coming from modes localized on the vinyl group. Peak (a) at $28 \mathrm{mV}$ corresponds to one of the torsional modes that is still well-defined for the planar configuration and also in-plane bending between the phenyl ring and the vinyl group. Peak (b) at $62 \mathrm{mV}$ involves out-of-plane bending in the phenyl ring and also another in-plane $\mathrm{C}-\mathrm{C}-\mathrm{C}$ bending mode. Peak (c) at $91 \mathrm{mV}$ contains the $6 \mathrm{a}$ and $6 \mathrm{~b}$ modes as well as a very strong contribution from out-of-plane $\mathrm{C}-\mathrm{H}$ bending on the vinyl group. Peak (d) at $144 \mathrm{mV}$ has strong contributions from in-plane and out-of-plane $\mathrm{C}-\mathrm{H}$ bending modes on both the aryl and vinyl groups. The sequence of peaks starting in the $200-230 \mathrm{mV}$ range involve $\mathrm{C}=\mathrm{C}$ stretching modes, including the ring stretching $8 \mathrm{a}$ and $8 \mathrm{~b}$ and the vinyl stretch, labeled (e) at $229 \mathrm{mV}$. Finally, the $416 \mathrm{mV}$ peak (f) has very strong contributions from all of the $\mathrm{C}-\mathrm{H}$ stretching modes.

The calculated IETS spectra for the OPVn series (Figure 1) are shown in Figures 5-8. Overall, the strongly active modes are similar to those of benzene and styrene with the exception of several additional low freqency skeletal bending modes in the $1-8 \mathrm{mV}$ range that cause adjacent phenyl rings to move in 

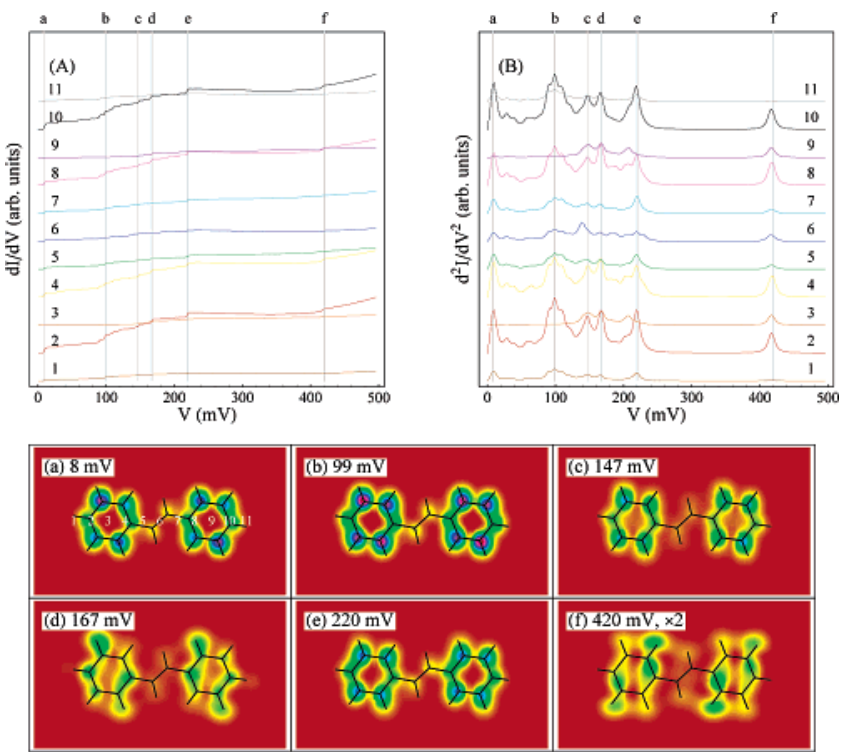

Figure 5. (A) and (B) calculated conductance and IETS intensities for OPV2 (stilbene) at different tip positions, depicted in (a). (a)-(f) Spatial maps of the most intense IETS peaks. See the caption in Figure 3 and text for more details.
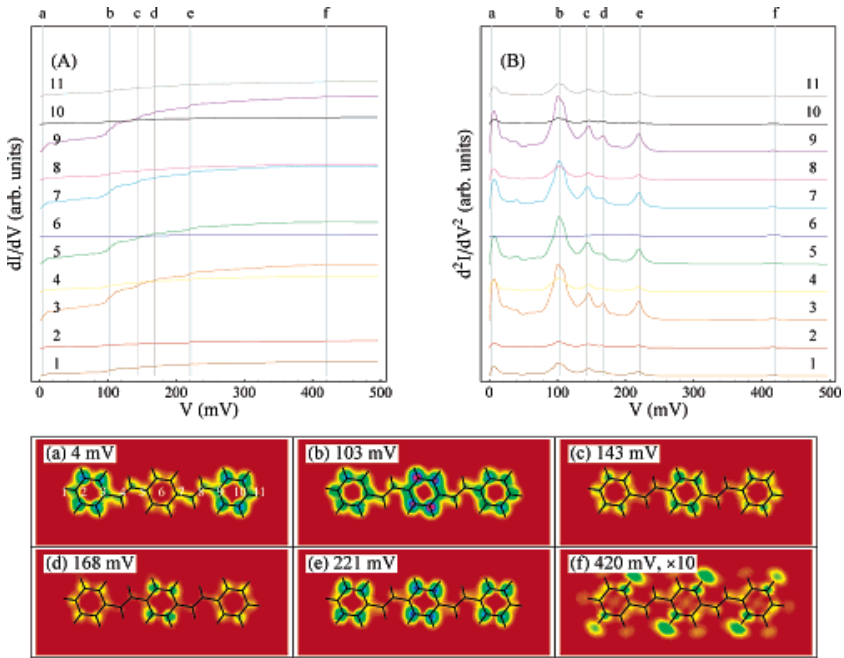

Figure 6. (A) and (B) calculated conductance and IETS intensities for OPV3 (distrylbenzene) at the different tip positions, depicted in (a). (a)-(f) spatial maps of the most intense IETS peaks. See the caption in Figure 3 and text for more details.

and out of the molecular plane relative to one another; this motion resembles standing wave type distortions across the entire carbon skeleton. These modes are labeled as (a) in Figures 5-8 and the corresponding spectral maps show that IETS intensity is distributed across the molecule with the largest intensity on the carbon atoms which are displaced out of the molecular plane. The OPVn molecules exhibit a broad and intense peak (b) centered around $100 \mathrm{mV}$ arising from several different vibrational modes. These include radial skeletal vibrations and out-of-plane skeletal and vibrations. Comparing the spatial maps for this peak, we see that the IETS signal for the smaller OPV2 and OPV3 molecules is strongest on phenyl rings but for OPV4 and OPV5 the intensity is enhanced on the vinyl groups. Peak (c) at $143 \mathrm{mV}$ corresponds to out-of-plane $\mathrm{C}-\mathrm{H}$ bending vibrations on the phenyl rings and is particularly intense for the larger OPV5 molecule. The in-plane $\mathrm{C}-\mathrm{H}$ bending modes on the phenyl rings give rise to a strong peak (d) at $168 \mathrm{mV}$. The spatial profiles of the IETS intensities share a similar structure with the exception of OPV 3 where the peak
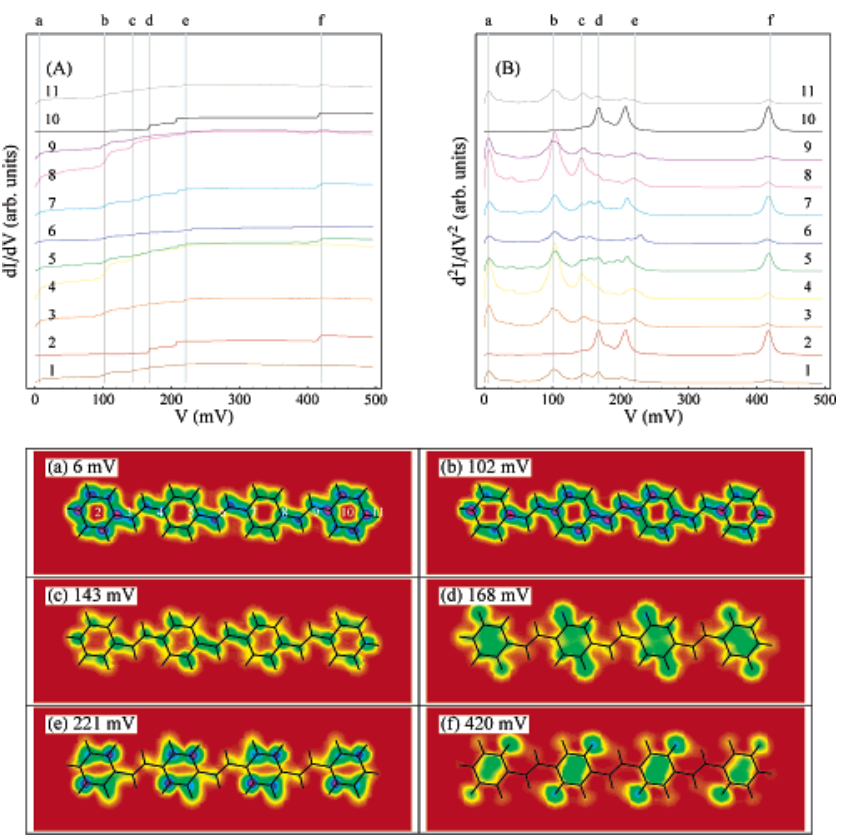

Figure 7. (a) and (b) calculated conductance and IETS intensities for OPV4 at the different tip positions, depicted in (c). (c)-(h) spatial maps of the most intense IETS peaks. See the caption in Figure 3 and text for more details.
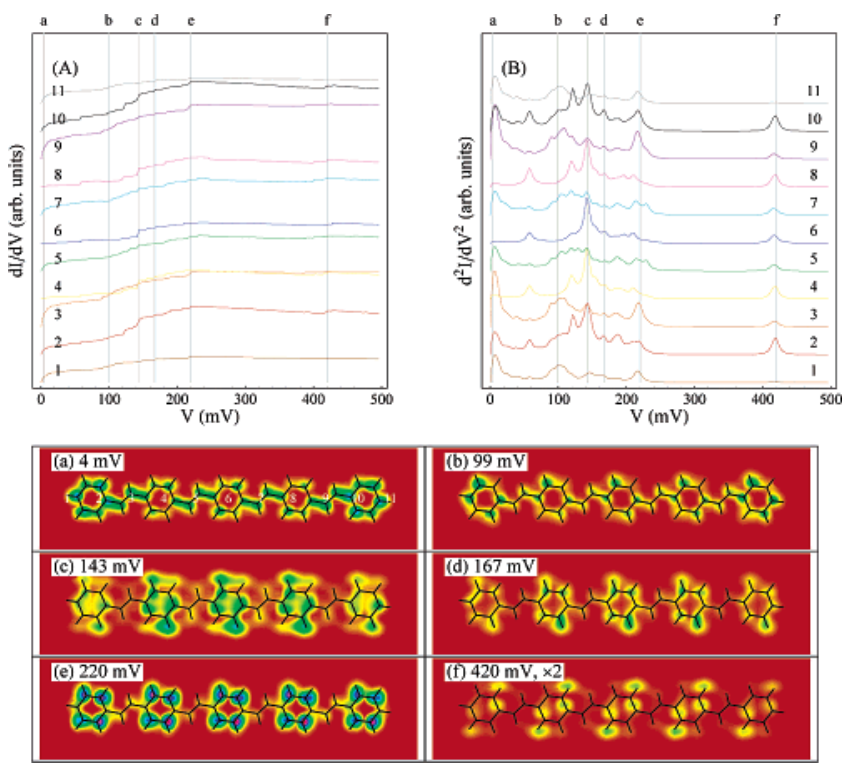

Figure 8. (A) and (B) calculated conductance and IETS intensities for OPV5 at the different tip positions, depicted in (a). (a)-(f) spatial maps of the most intense IETS peaks. See the caption in Figure 3 and text for more details.

is much less intense compared to those of the other molecules. Peak (e) at $220 \mathrm{mV}$ corresponds to ring stretching modes and the IETS intensity sharply increases over the phenyl carbon atoms. Peak (f) at $420 \mathrm{mV}$ contains contributions from all of the different $\mathrm{C}-\mathrm{H}$ stretching modes, but the largest contribution to the IETS intensity comes from hydrogen atoms on the phenyl rings. This portion of the spectrum is much less intense for OPV3 compared to the other of the chromophores; however, all of the spatial maps for this peak have similar patterns.

Kushmerick et al. ${ }^{10}$ have measured the IET spectrum of a thiolated OPV3 monolayer sandwiched between gold electrodes in a cryogenic tunnel junction. It shows peaks associated with aryl $\mathrm{C}-\mathrm{H}$ out-of-plane bending and longitudinal modes (i.e., parallel to the direction of current) $18 \mathrm{a}, 15,8 \mathrm{a}$, and $v_{\mathrm{C}=\mathrm{C}}$. Very 


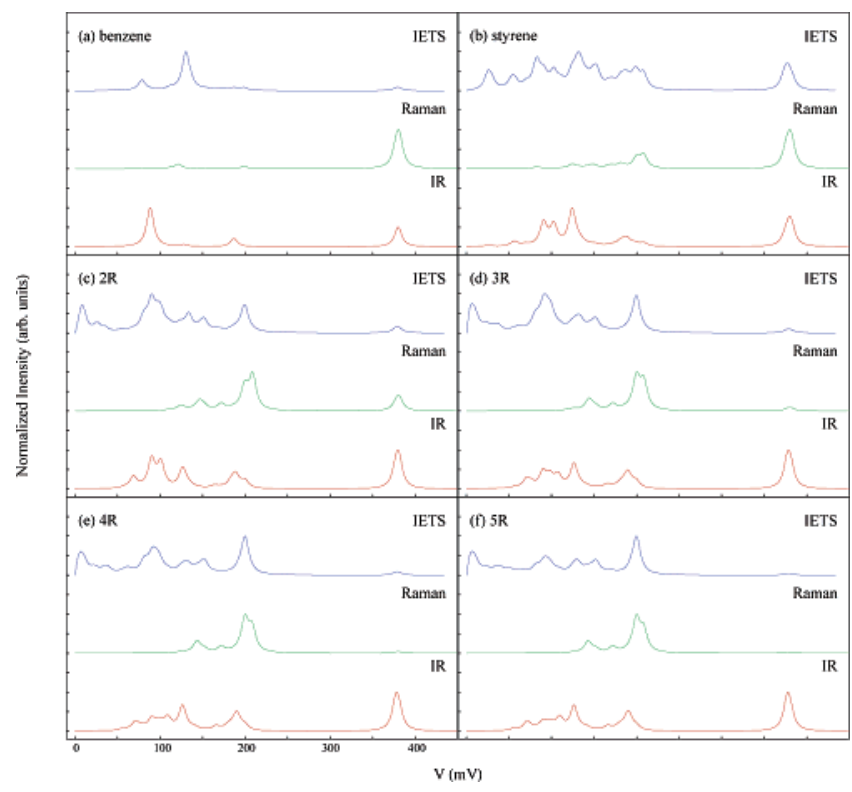

Figure 9. Comparison of spatially averaged (blue) IETS, (green) Raman, and (red) IR spectra for (a) benzene, (b) styrene, and (c)-(f) $\mathrm{OPV} n$ molecules. The IETS signal does not obey the symmetry selection rules of either IR or Raman spectroscopy.

recently, Troisi and Ratner ${ }^{77}$ have calculated the IET spectra for OPV3 in the molecule wire configuration with two mock electrodes located at both ends of the molecule and fixed parameters representing the coupling to the molecular orbitals. They use a perturbative method to calculate the inelastic contributions to electronic Greens functions; their approach gives remarkably good agreement of relative peak intensities with experiment.

Figure 9 compares the spatially averaged IETS intensities of benzene, styrene, and the OPV $n$ series to their corresponding IR and Raman intensities. The IR and Raman intensities for each mode are determined from quantum chemistry calculations and we have added a small Lorentzian line width $(0.01 \mathrm{eV})$ to simulate the line shape. This comparison illustrates that the symmetry selection rules that are familiar from IR and Raman spectroscopy do not apply to single molecule IETS.

Paracyclophane and DBP. The family of DBP molecules are shown in Figure 2. For paracyclophane, it is generally agreed that the benzene rings are slightly twisted relative to one another with $D_{2}$ symmetry. As noted in ref 78, restricted Hartree-Fock methods, which lack electron correlation effects beyond exchange, predict the eclipsed $D_{2 h}$ geometry. Our HF/3-21G calculations also converge to the eclipsed structure and because no imaginary frequencies were found in the normal mode spectrum, we use the $D_{2 h}$ structure. In Figure 10A,B we show the inelastic conductance profiles and IETS spectra for [2.2]paracyclophane with the tip orbital centered at different positions in the plane $1 \AA$ above and parallel to two benzene "decks"; see Figure 10a. The $9 \mathrm{mV}$ peak corresponds to several lowfrequency bending modes that distort the paracyclophane skeleton; the most strongly coupled mode involves symmetric rotation of the alkyl bridge groups which cause the two decks to slide back and forth across one another. Peak (b) at $71 \mathrm{mV}$ involves another type of deformation where the benzene rings tilt in to and out of the viewing plane. Peaks (c) at $100 \mathrm{mV}$ and (d) at $139 \mathrm{mV}$ correspond to out-of-plane skeletal and $\mathrm{C}-\mathrm{H}$ bending modes on the phenyl groups, respectively. The strong peak (e) at $220 \mathrm{mV}$ involves several ring stretching modes. Peak (f) at $414 \mathrm{mV}$ corresponds to the $\mathrm{C}-\mathrm{H}$ stretching modes of the aryl hydrogens; the bridge hydrogens are not as strongly coupled.
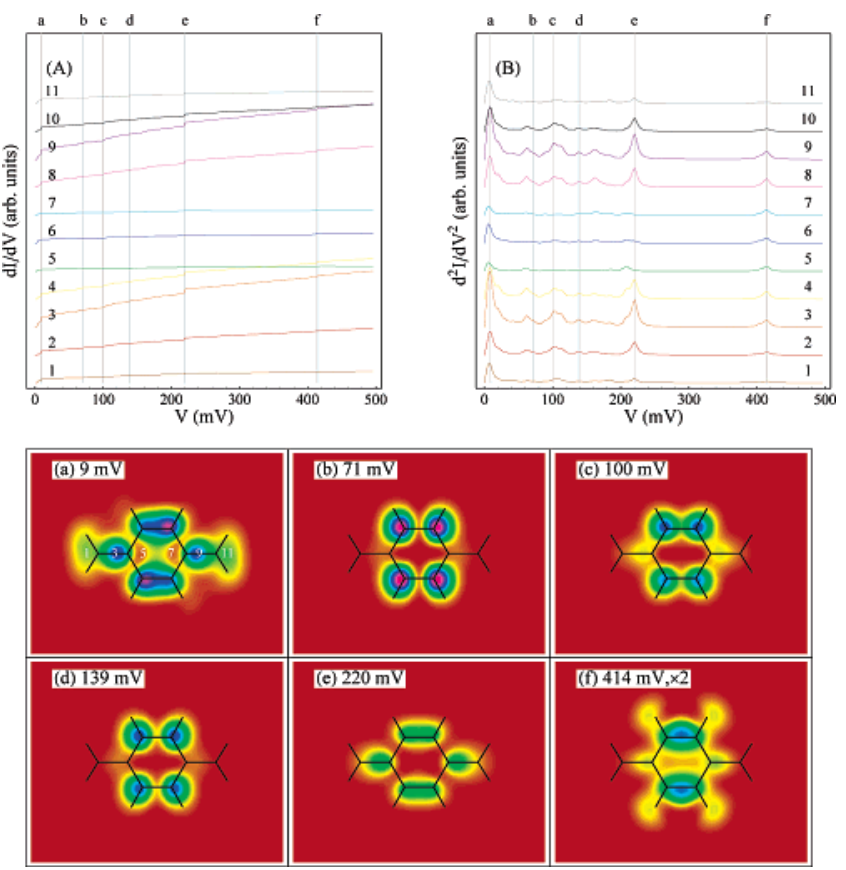

Figure 10. (A) and (B) calculated conductance and IETS intensities for paracyclophane at the different tip positions, depicted in (a). (a)(f) spatial maps of the most intense IETS peaks. The images are sliced at $1 \AA$ above and parallel to the two $\pi$-stacked benzene decks. See the caption in Figure 3 and text for more details.

Next we turn to the larger DBP molecules. The potential energy surfaces for these molecules have multiple local minima and transition states ${ }^{79}$ and the optimized geometry depends on starting geometry. This is due primarily to flatness of the potential energy surface near the minimum energy dihedral angle about the single $\mathrm{C}-\mathrm{C}$ bonds linking the vinyl and phenyl groups. Similar issues have been examined extensively with OPV $n$ molecules, where it has been noted that different quantum chemistry models predict contradictions regarding molecular planarity. ${ }^{80}$ This problem is exacerbated in the DBPs where the styryl groups on the two benzene decks are held in close proximity, thus introducing nonbonded interactions that further complicate the potential. In our HF/3-21G calculations we enforced a planar configuration of the dihedral angles between the vinyl and phenyl groups. For each molecule, the paracyclophane core is slightly twisted due to the additional strain imposed by the styryl groups. In Figures $11-13$ we show the calculated IETS resonances. The presence of the distyrylbenzene groups dramatically increases the complexity of the spectra compared to those of paracyclophane. For example, contributions from the low-frequency bending modes associated with the paracyclophane core and the styryl arms overlap in the vicinity of peak (a) at $5-10 \mathrm{mV}$. The spatial profiles of these peaks show that the distribution of the IETS intensity over the molecule is similar to that for the low-frequency peaks in the OPV $n$ chromophores. The lower styryl arms do not contribute to the spatial pattern because the tip orbital does not significantly overlap with the molecular oribtals in that region of space. Similar to the OPVn molecules, peaks (b) at $99 \mathrm{mV}$ and (c) at $140 \mathrm{mV}$ correspond to out-of-plane skeletal and $\mathrm{C}-\mathrm{H}$ bending on the phenyl rings, respectively. Peak (d) at $167 \mathrm{mV}$ corresponds to the in-plane aryl $\mathrm{C}-\mathrm{H}$ bending modes and peak (e) at $214 \mathrm{mV}$ is due to ring stretching. For cis-2Rd and $3 \mathrm{Rd}$, the local structure of the spatially resolved IETS intensity for peaks (b)-(e) is more or less the same on the paracyclophane and styryl group(s). For trans-2Rd, however, the IETS intensity is much stronger on the core compared to that on the styryl arm. 

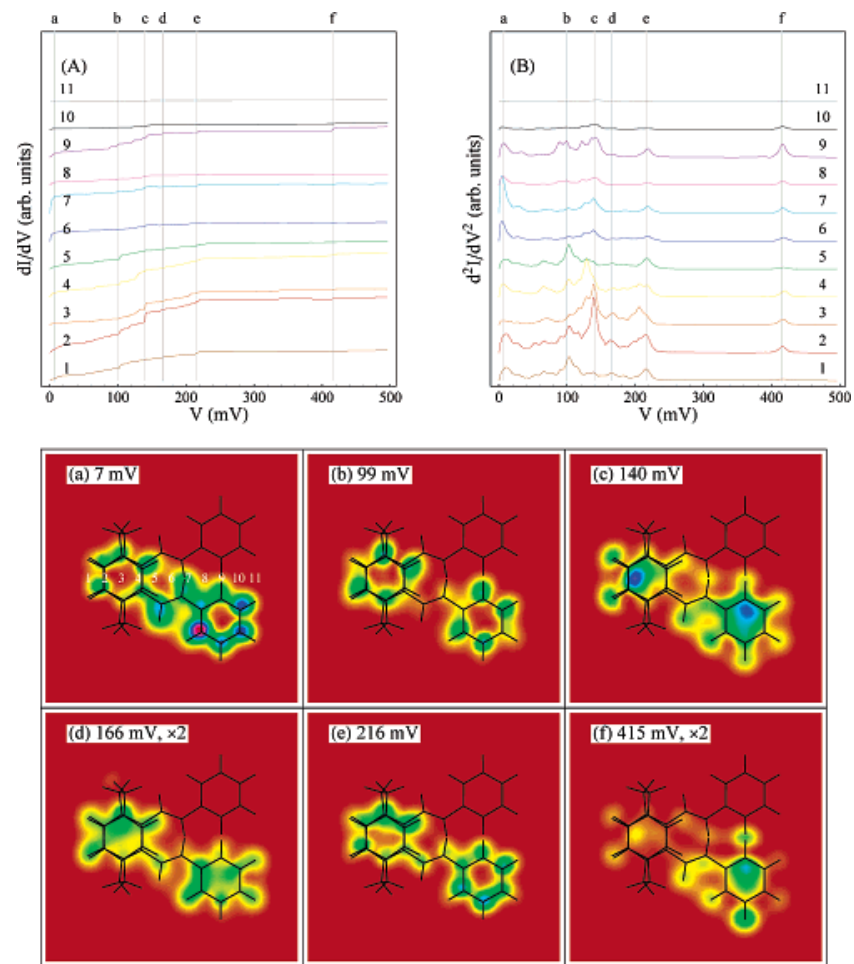

Figure 11. (A) and (B) calculated conductance and IETS intensities for cis-2Rd at the different tip positions, depicted in (a). (a)-(f) spatial maps of the most intense IETS peaks. The images are sliced at $1 \AA$ above and parallel to the two $\pi$-stacked benzene decks. See the caption in Figure 3 and text for more details.
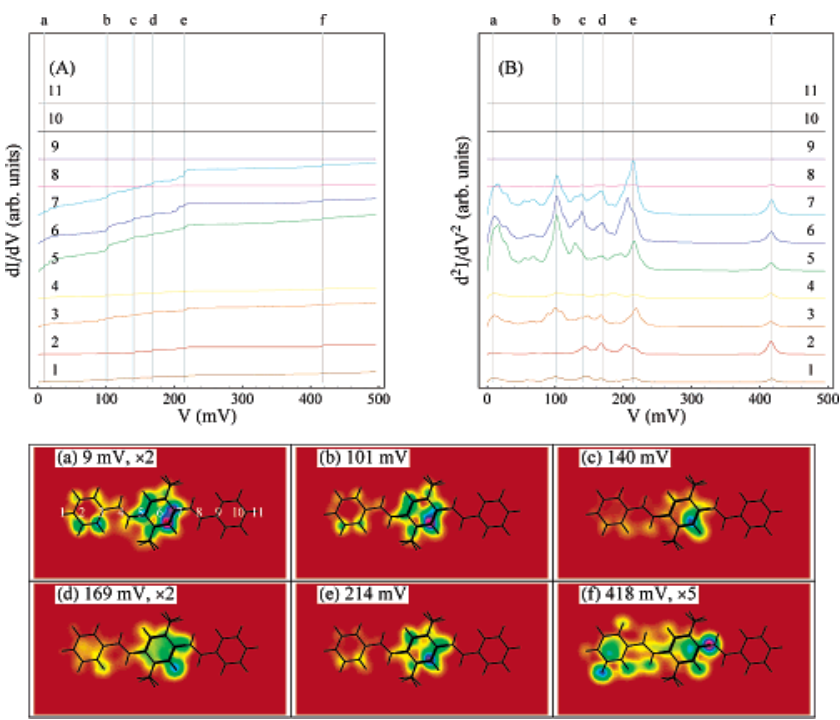

Figure 12. (A) and (B) calculated conductance and IETS intensities for trans-2Rd at the different tip positions, depicted in (a). (a)-(f) spatial maps of the most intense IETS peaks. The images are sliced at $1 \AA$ above and parallel to the two $\pi$-stacked benzene decks. See the caption in Figure 3 and text for more details.

Finally peak (f) at $415 \mathrm{mV}$ coresponds to $\mathrm{C}-\mathrm{H}$ stretching modes localized on different parts of the molecules. The aryl hydrogens on the distyryl groups have the strongest coupling to the tunneling current. It is interesting to note the differences between the IETS intensities of the cis- and trans-2Rd isomers. Compared to cis-2Rd, the intensity of the torsional modes in the trans$2 \mathrm{Rd}$ are suppressed relative to the bending and stretching modes localized on the vinyl and phenyl groups. Such differences are not seen in the calculated IR and Raman lines (see Figure 14), which are nearly the same for the pair of isomers.
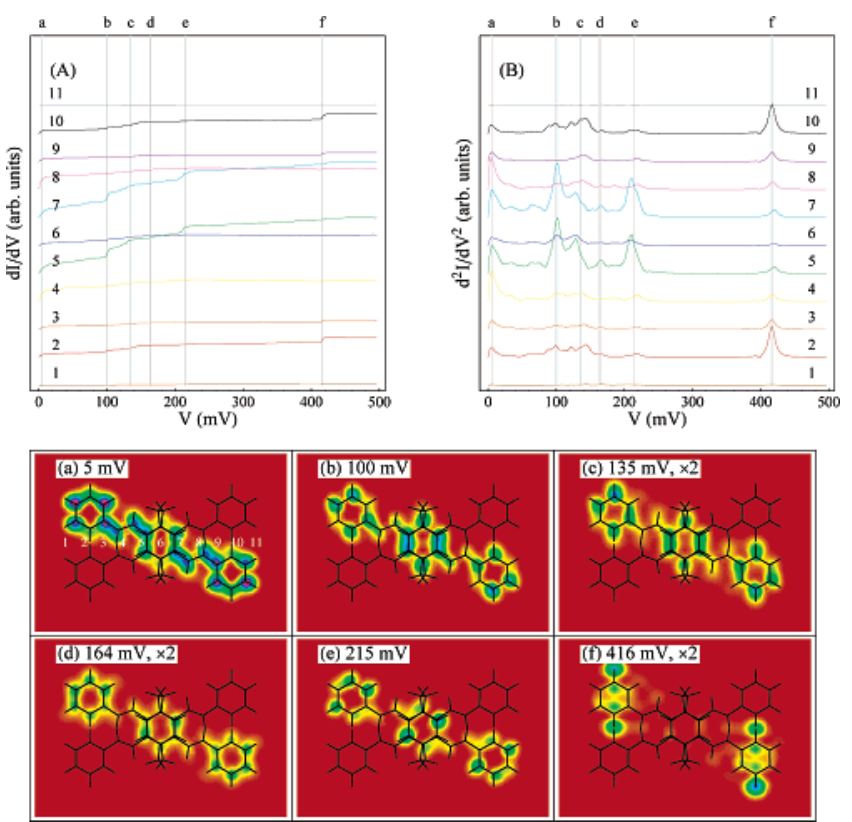

Figure 13. (A) and (B) calculated conductance and IETS intensities for $3 \mathrm{Rd}$ at the different tip positions, depicted in (a). (a)-(f) spatial maps of the most intense IETS peaks. The images are sliced at $1 \AA$ above and parallel to the two $\pi$-stacked benzene decks. See the caption in Figure 3 and text for more details.

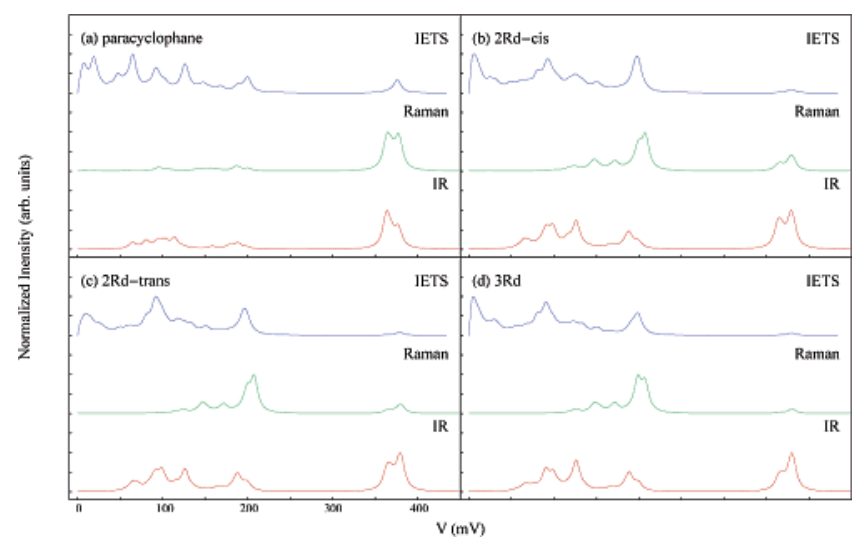

Figure 14. Comparison of calculated (blue) IETS, (green) Raman, and (red) IR spectra for (a) paracyclophane and (b) - (d) distyrylbenzene molecules. The IETS signal does not obey the symmetry selection rules of either IR or Raman spectroscopy.

Very recently, Seferos et al. ${ }^{12}$ compared the conductance of thiolated OPV3 and trans-2Rd monolayers on gold electrodes. They found that the conductivity per molecule is roughly same between the two different species; however, they have not reported IET measurements for trans-2Rd. Liu et al. ${ }^{35}$ have used low-temperature STM-IETS to study the inelastic vibrational resonances of a dimethyl-amino derivative of 3Rd (DMAS-PCP) on a $\mathrm{NiAl}(110)$ surface. Five peaks at 25, 40, 60, 180, and 360 $\mathrm{mV}$ were resolved and the relative intensities of the peaks show significant variation as a function of the tip's position. In Figure 15 we compare one of the experimental IETS curves for DMASPCP to one of the calculated spectra for $3 \mathrm{Rd}$. Both spectra are normalized such that the largest peak has unit intensity and we have scaled the bias values of calculated spectrum by 0.9085 to compensate for the overestimation of vibrational transition energies in our HF calculations. Because we have used a gas phase molecular structure and neglected the influence of the metal substrate, the calculated maps do not quantitatively reproduce the experimental STM-IETS images. Nevertheless, our results are useful for assigning the vibrational modes that 


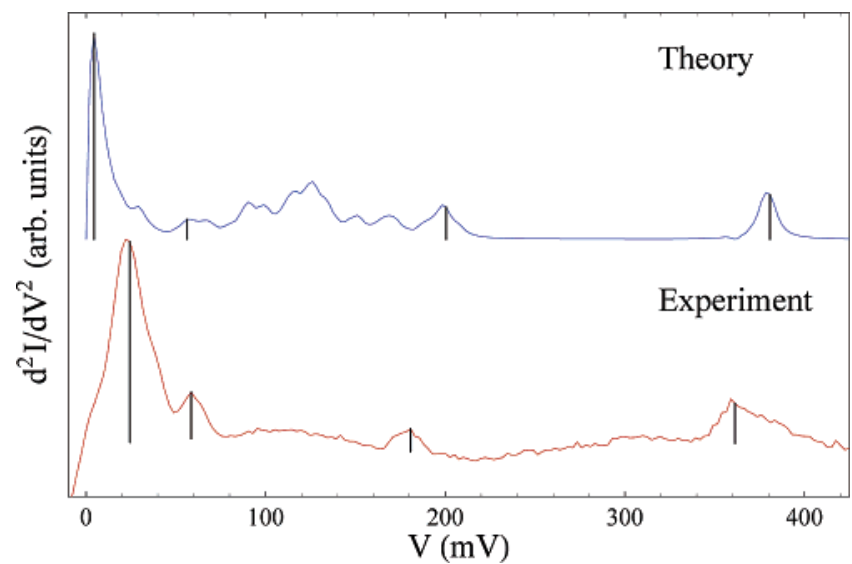

Figure 15. Comparison of (red) experimental and (blue) calculated IETS spectra for the 3Rd molecule. The intensities of the peaks are marked by vertical black lines and compared in Table 1 .

TABLE 1: Comparison of the Experimental and Calculated IETS Spectral Positions and Relative Peak Intensities for the Inelastic Resonances of 3Rd

\begin{tabular}{|c|c|c|c|}
\hline \multicolumn{2}{|c|}{ experiment } & \multicolumn{2}{|c|}{ theory } \\
\hline $\mathrm{mV}$ & $I^{\prime \prime} / I_{\max }^{\prime \prime}$ & $\mathrm{mV}$ & $I^{\prime \prime} / I_{\max }^{\prime \prime}$ \\
\hline 24 & 1.00 & 5 & 1.00 \\
\hline 61 & 0.19 & 56 & 0.10 \\
\hline 181 & 0.11 & 200 & 0.16 \\
\hline 361 & 0.19 & 381 & 0.22 \\
\hline
\end{tabular}

contribute to the peak structure and comparing the relative peak intensities. Our calculations suggest that the experimental peaks at 25,40 , and $60 \mathrm{mV}$ involve low-frequency bending modes associated with torsional motion in the distyrylbenzene arms and paracyclophane core. These modes are distributed over the entire carbon skeleton of the molecule but can involve subtle distortions in paracyclophane core that cause the benzene decks to slide and twist relative to one another. The $180 \mathrm{mV}$ peak is likely due to $\mathrm{C}-\mathrm{H}$ bending motion in the bridge groups with additional contributions from in-plane bending that extends over the styryl arms. All the $\mathrm{C}-\mathrm{H}$ stretching modes of $3 \mathrm{Rd}$ contribute to the IETS with roughly equal strength. The bridge $\mathrm{C}-\mathrm{H}$ stretch modes are spectrally distinct and have a lower energy than the aryl and vinyl modes; this can be useful to identify the position of the bridge groups in the experimental IETS spatial map by tuning the voltage to the red side of the $\mathrm{C}-\mathrm{H}$ stretching band. In Table 1 we compare the peak positions and relative intensities of these resonances. Our calculated results for $3 R d$ qualitatively reproduce the relative intensities observed in the experimental STM-IETS spectrum for DMAS-PCP.

\section{Summary}

We have calculated the electron-phonon coupling in OPV $n$ and DBPs and used a perturbative expansion of the molecular electronic Greens function to simulate vibrational resonances in STM-IETS. Our calculations provide physical insight with regard to which internal molecular vibrations will likely contribute to the inelastic current through a single molecule. This is confirmed by comparison with experiments. IET signals do not have strict symmetry selection rules. Instead, all vibrational modes contribute to the tunneling current but some modes have a higher propensity for stronger inelastic tunneling that depends on the precise details of the coupling between the molecular electronic and nuclear degrees of freedom.

Acknowledgment. We gratefully acknowledge the support of the National Science Foundation (CHE 0446555, ECCC-
0406750, and CHE 5533162). C.S. is a Scientific Research Worker of the Belgian National Fund for Scientific Research (FNRS). We thank M. Persson, M. A. Ratner and O. Hod for stimulating discussion and also thank M. J. Frisch for pointing out to us the capabilities of the CPHF features in Gaussian 03.

\section{References and Notes}

(1) Jaklevic, R. C.; Lambe, J. Phys. Rev. Lett. 1966, 17, 1139.

(2) Hansma, P. K. Phys. Rep. 1977, 30, 145.

(3) Hipps, K. W.; Mazur, U. J. Phys. Chem. 1993, 97, 7803.

(4) Nitzan, A. Annu. Rev. Phys. Chem. 2001, 52, 681.

(5) Reed, M. A.; Zhou, C.; Muller, C. J.; Burgin, T. P.; Tour, J. M. Science 1997, 278, 252.

(6) Fan, F. F.; Yang, J.; Dirk, S. M.; Price, D. W.; Kosynkin, D.; Tour,

J. M.; Bard, A. J. J. Am. Chem. Soc. 2001, 123, 2454.

(7) Chen, J.; Reed, M. A. Chem. Phys. 2002, 281, 127.

(8) Wang, W.; Lee, T.; Kretzchmar, I.; Reed, M. A. Nano Lett. 2004, $4,643$.

(9) Wang, W.; Lee, T.; Reed, M. A. J. Phys. Chem. B 2004, 108, 18398.

(10) Kushmerick, J. G.; Lazorcik, J.; Patterson, C. H.; Shashidhar, R Seferos, D. S.; Bazan, G. C. Nano Lett. 2004, 4, 639.

(11) Hallbäck, A. S.; Oncel, N.; Huskens, J.; Zandvliet, H. J. W.; Poelsema, B. Nano. Lett. 2004, 4, 2393.

(12) Seferos, D. S.; Trammell, S. A.; Bazan, G. C.; Kushmerick, J. G. Proc. Natl. Acad. Sci. U.S.A. 2005, 102, 8821.

(13) Smit, R. H. M.; Noat, Y.; Untiedt, C.; Lang, N. D.; van Hemert, M. C.; van Ruitenbeek, J. M. Nature 2002, 419, 906.

(14) Kubatkin, S.; Danilov, A.; Hjort, M.; Cornil, J.; Brédas, J. L.; Hansen, N. S.; Hedegoard, P.; Bjornholm, T. Nature 2003, 425, 698

(15) Elbing, M.; Ochs, R.; Koentopp, M.; Fischer, M.; von Hänisch, C.; Weigend, F.; Evers, F.; Weber, H. B.; Mayor, M. Proc. Natl. Acad. Sci. U.S.A. 2005, 102, 8815.

(16) Pascual, J. I.; Jackiw, J. J.; Song, Z.; Weiss, P. S.; Conrad, H.; Rust, H.-P. Surf. Sci. 2002, 502, 1.

(17) Sykes, E. C. H.; Mantooth, B. A.; Han, P.; Donhauser, Z. J.; Weiss, P. S. J. Am. Chem. Soc. 2005, 127, 7255.

(18) Hla, S.-W.; Bartels, L.; Meyer, G.; Rieder, K.-H. Phys. Rev. Lett. 2000, 85, 2777.

(19) Gaudisoso, J.; Lee, H. J.; Ho, W. J. Am. Chem. Soc. 1999, 121, 8479 .

(20) Kim, Y.; adnd M. Kawai, T. K. Phys. Rev. Lett. 2002, 89, 126104.

(21) Stipe, B. C.; Rezaei, M. A.; Ho, W. Science 1998, 279, 1907.

(22) Komeda, T.; Kim, Y.; Kawai, M.; Persson, B. N. J.; Ueba, H. Science 2002, 295, 2055.

(23) Komeda, T.; Kim, Y.; Kawai, M. Surf. Sci. 2002, 502, 12.

(24) Alavi, S.; Larade, B.; Taylor, J.; Guo, H.; Seideman, T. Chem. Phys. 2002, 281, 293.

(25) Seideman, T. J. Phys.: Condens. Matter 2003, 15, 521.

(26) Stipe, B. C.; Rezaei, M. A.; Ho, W. Phys. Rev. Lett. 1998, 81, 1263.

(27) Stipe, B. C.; Rezaei, M. A.; Ho, W. Rev. Sci. Instrum. 1999, 70, 137.

(28) Wallis, T. M.; Chen, X.; Ho, W. J. Chem. Phys. 2000, 113, 4837.

(29) Lauhon, L. J.; Ho, W. Phys. Rev. Lett. 2000, 85, 4566.

(30) Hahn, J. R.; Lee, H. J.; Ho, W. Phys. Rev. Lett. 2000, 85, 1914

(31) Pascual, J. I.; Jackiw, J. J.; Song, Z.; Weiss, P. S.; Conrad, H.; Rust, H.-P. Phys. Rev. Lett. 2001, 86, 1050.

(32) Pascual, J. I.; Gómez-Herrero, J.; Sánches-Portal, D.; Rust, H. P. J. Chem. Phys. 2002, 117, 9531.

(33) Vitali, L.; Burghard, M.; Schneider, M. A.; Liu, L.; Wu, S. Y.; Jayanthi, C. S.; Kern, K. Phys. Rev. Lett. 2004, 93, 136103.

(34) Grobis, M.; Khoo, K. H.; Yamachika, R.; Lu, X.; Nagaoka, K.; Louie, S. G.; Crommie, M. F.; Kato, H.; Shinohara, H. Phys. Rev. Lett. 2005, 94, 136802

(35) Liu, N.; Silien, C.; Ho, W.; Maddox, J. B.; Mukamel, S.; Liu, B.; Bazan, G. C. Manuscript in preparatation, 2006.

(36) Ho, W. J. Chem. Phys. 2002, 117, 11033

(37) Galperin, M.; Ratner, M. A.; Nitzan, A. Nano Lett. 2005, 5, 125.

(38) Gaudioso, J.; Lauhon, L. J.; Ho, W. Phys. Rev. Lett. 2000, 85, 1918

(39) Gaudisoso, J.; Ho, W. J. Am. Chem. Soc. 2001, 123, 10095

(40) Guisinger, N. P.; Yoder, N. L.; Hersam, M. C. Proc. Natl. Acad. Sci. U.S.A. 2005, 102, 8838

(41) Hliwa, H.; Joachim, C. Phys. Rev. B 2002, 65, 085406

(42) Segal, D.; Nitzan, A. J. Chem. Phys. 2002, 117, 3915.

(43) Segal, D.; Nitzan, A.; Hänggi, P. J. Chem. Phys. 2003, 119, 6840

(44) Chen, Y.; Zwolak, M.; Ventra, M. D. Nano Lett. 2004, 5, 621.

(45) Silien, C.; Liu, N.; Ho, W.; Maddox, J. B.; Mukamel, S.; Liu, B.; Bazan, G. C. Manuscript in preparatation, 2006.

(46) Burroughes, J. H.; Bradley, D. D. C.; Brown, A. R.; Marks, R. N.; Mackay, K.; Friend, R. H.; Burns, P. L.; Holmes, A. B. Nature 1990, 347 539. 
(47) Bazan, G. C.; Oldham, W. J.; Lachicotte, R. J.; Tretiak, S.; Chernyak, V.; Mukamel, S. J. Am. Chem. Soc. 1998, 120, 9188.

(48) Caroli, C.; Combescot, R.; Nozieres, P.; Saint-James, D. J. Phys. C: Solid State Phys. 1972, 5, 21.

(49) Tikhodeev, S.; Natario, M.; Makoshi, K.; Mii, T.; Ueba, H. Surf. Sci. 2001, 493, 63.

(50) Mii, T.; Tikhodeev, S.; Ueba, H. Surf. Sci. 2002, 502, 26.

(51) Mii, T.; Tikhodeev, S. G.; Ueba, H. Phys. Rev. B 2003, 68, 205406.

(52) Xue, Y.; Datta, S.; Ratner, M. A. J. Chem. Phys. 2001, 115, 4292.

(53) Damle, P.; Ghosh, A. W.; Datta, S. Chem. Phys. 2002, 281, 171.

(54) Xue, Y.; Datta, S.; Ratner, M. A. Chem. Phys. 2002, 281, 151.

(55) Galperin, M.; Ratner, M. A.; Nitzan, A. Nano Lett. 2004, 4, 1605.

(56) Galperin, M.; Ratner, M. A.; Nitzan, A. J. Chem. Phys. 2004, 121 11965

(57) Galperin, M.; Nitzan, A.; Ratner, M. A.; Stewart, D. R. J. Phys. Chem. B 2005, 109, 8519.

(58) Asai, Y. Phys. Rev. Lett. 2004, 93, 246102.

(59) Lorente, N.; Persson, M. Phys. Rev. Lett. 2000, 85, 2997.

(60) Lorente, N.; Persson, M.; Lauhon, L. J.; Ho, W. Phys. Rev. Lett. 2001, 86, 2593

(61) Datta, S. Electronic Transport in Mesoscopic Systems; Cambridge University Press: Cambridge, U.K., 1997.

(62) Haug, H.; Jauho, A.-P. Quantum Kinetics in Transport and Optics of Semiconductors; Springer: Germany, 1996.

(63) Harbola, U.; Maddox, J. B.; Mukamel, S. Phys. Rev. B 2006, in press.

$$
99 .
$$

(64) Kirkegaard, C.; Kim, T. K.; Hofmann, P. New J. Phys. 2005, 7,

(65) Frisch, M. J.; Trucks, G. W.; Schlegel, H. B.; Scuseria, G. E.; Robb, M. A.; Cheeseman, J. R.; Montgomery, J. A., Jr.; Vreven, T.; Kudin, K. N.; Burant, J. C.; Millam, J. M.; Iyengar, S. S.; Tomasi, J.; Barone, V.; Mennucci, B.; Cossi, M.; Scalmani, G.; Rega, N.; Petersson, G. A.; Nakatsuji, H.; Hada, M.; Ehara, M.; Toyota, K.; Fukuda, R.; Hasegawa, J.;
Ishida, M.; Nakajima, T.; Honda, Y.; Kitao, O.; Nakai, H.; Klene, M.; Li, X.; Knox, J. E.; Hratchian, H. P.; Cross, J. B.; Bakken, V.; Adamo, C.; Jaramillo, J.; Gomperts, R.; Stratmann, R. E.; Yazyev, O.; Austin, A. J.; Cammi, R.; Pomelli, C.; Ochterski, J. W.; Ayala, P. Y.; Morokuma, K.; Voth, G. A.; Salvador, P.; Dannenberg, J. J.; Zakrzewski, V. G.; Dapprich, S.; Daniels, A. D.; Strain, M. C.; Farkas, O.; Malick, D. K.; Rabuck, A. D.; Raghavachari, K.; Foresman, J. B.; Ortiz, J. V.; Cui, Q.; Baboul, A. G.; Clifford, S.; Cioslowski, J.; Stefanov, B. B.; Liu, G.; Liashenko, A.; Piskorz, P.; Komaromi, I.; Martin, R. L.; Fox, D. J.; Keith, T.; Al-Laham, M. A.; Peng, C. Y.; Nanayakkara, A.; Challacombe, M.; Gill, P. M. W.; Johnson, B.; Chen, W.; Wong, M. W.; Gonzalez, C.; Pople, J. A. Gaussian 03, revision C.02; Gaussian, Inc.: Wallingford, CT, 2004.

(66) Taylor, J.; Guo, H.; Wang, J. Phys. Rev. B 2001, 63, 245407.

(67) Tersoff, J.; Hamann, D. R. Phys. Rev. Lett. 1983, 50, 1998.

(68) Tersoff, J.; Hamann, D. R. Phys. Rev. B 1985, 31, 805.

(69) Sergueev, N.; Roubtsov, D.; Guo, H. Phys. Rev. Lett. 2005, 95, 146803.

(70) Wilson, E. B. Phys. Rev. 1934, 45, 706.

(71) Varsányi, G. Assignments for vibrational spectra of seven hundred benzene derivatives; John Wiley \& Sons: New York, 1974.

(72) Lauhon, L. J.; Ho, W. J. Phys. Chem. A 2000, 104, 2436.

(73) Lauhon, L.; Ho, W. Surf. Sci. 2000, 451, 219

(74) Komeda, T.; Kim, Y.; Fujita, Y.; Sainoo, Y.; Kawai, M. J. Chem. Phys. 2004, 120, 5347

(75) Asai, Y. Phys. Rev. Lett. 2005, 94, 099901.

(76) Choi, C. H.; Kertesz, M. J. Phys. Chem. A 1997, 101, 3823.

(77) Troisi, A.; Ratner, M. A. Phys. Rev. B 2005, 72, 033408.

(78) Walden, S. E.; Glatzhofer, D. T. J. Phys. Chem. A 1997, 101, 8233.

(79) Ruseckas, A.; Namdas, E. B.; Lee, J. Y.; Mukamel, S.; Wang, S.; Bazan, G. C.; Sundström, V. J. Phys. Chem. A 2003, 107, 8029.

(80) Masunov, A.; Tretiak, S.; Hong, J. W.; Liu, B.; Bazan, G. C. J. Chem. Phys. 2005, 122, 223505. 